\title{
Risk Factors of Polymicrobial Bloodstream Infections in Pediatric Intensive Care Unit Patients
}

\author{
Ali A. Abdo', Ehab A. Albanna ${ }^{1}$, Salah E. Ebrahim², Dina S. Mahmoud ${ }^{* 1}$ \\ Departments of ${ }^{1}$ Pediatrics and ${ }^{2}$ Clinical Pathology, Faculty of Medicine, Zagazig University, Egypt \\ *Corresponding author: Dina S. Mahmoud, Mobile: (+20) 01151413303, E-Mail: raghadrami779@ yahoo.com
}

\begin{abstract}
Background: Sepsis continues to present a significant problem in the pediatric intensive care unit (PICU) worldwide. Sepsis in pediatric infants is an important cause of morbidity and mortality. The risk of sepsis increases with decreasing chronological age and weight.

Objective: To identify of the risk factors of polymicrobial BSIs in pediatric patients with intravascular devices, via conducting the objectives, which were evaluating the frequency of monomicrobial and polymicrobial BSIs in pediatric patients. Patients and methods: This is observational cross sectional study that was conducted in at PICU, General Zagazig Hospital, carried on 90 pediatric patients who were divided into 4 groups: group (I) oncology patients, group (II) cardiac patients, group (III) gastrointestinal (GIT) patients and group (IV) miscellaneous patients.

Results: Our results showed that $50 \%$ of polymicrobial infection patients had cardiac disease, $25 \%$ had gastrointestinal disease. $25 \%$ had renal failure. $100 \%$ of the cardiac patients and the gastrointestinal patients had respiratory cause of admission to PICU.

Conclusion: Steroids given to the patients, endotracheal intubation, central venous catheter (CVC), congenital heart diseases (CHD) and $\mathrm{H} 2$ blocker were the most common risk factors for polymicrobial infection in PICU.
\end{abstract}

Keywords: BSI, Polymicrobial, Monomicrobial, PICU.

\section{INTRODUCTION}

Healthcare-associated bloodstream infections (BSIs) are an important cause of increased healthcare costs, morbidity, and mortality ${ }^{(\mathbf{1})}$. Although essential in medical practice for patients in whom frequent vascular access is required, the use of central venous catheters (CVCs) is the leading risk factor for BSI. Seventy percent of healthcare-associated BSIs are estimated to occur among patients with CVCs ${ }^{(2)}$. Indications for central venous catheter (CVC) use are diverse, varying from administration of chemotherapy or antibiotics to the delivery of long-term parenteral nutrition ${ }^{(3)}$. Children with CVCs are at high risk of infectious complications associated with the presence of the catheter itself, as well the child's underlying conditions ${ }^{(4)}$.

The incidence of individual monomicrobial pathogens in BSIs have been studied identifying different risk factors, such as age, underlying disease, type of catheter, and immune status, using continuous positive airway pressure (CPAP) and mechanical ventilator ${ }^{(5)}$. However, epidemiological data about the incidence and risk factors of catheter-related BSIs caused by polymicrobial infections are scarce ${ }^{(6)}$.

Catheter-related BSIs are most commonly caused by Gram-positive skin flora. Catheter-related BSIs due to Gram-negative organisms are less common. Only $10 \%$ of pediatric healthcareassociated BSIs are polymicrobial ${ }^{(7)}$. The mortality rate for polymicrobial BSI may approach twice that for monomicrobial BSI. Polymicrobial BSIs are also problematic for clinicians, because appropriate treatment frequently requires multiple broadspectrum antibiotics ${ }^{(8)}$.
Additionally, antibiotic choices rely on prompt identification of all pathogens, and this takes more time and may be costlier than identification of a single pathogen. Furthermore, less is known about which patients are at risk for these infections ${ }^{(9)}$.The aim of the study was to identify of the risk factors of polymicrobial BSIs in pediatric patients with intravascular devices, via conducting the objectives, which were evaluating the frequency of monomicrobial and polymicrobial BSIs in pediatric patients, identify the clinical risk factors associated with the occurrence of polymicrobial infections in an inpatient setting and comparing clinical outcomes between pediatric patients with polymicrobial BSI and those with monomicrobial BSI.

\section{PATIENTS AND METHODS}

This is observational cross-sectional study that was conducted in PICU, General Zagazig Hospital, and carried on 90 pediatric patients.

\section{Target population and criteria for inclusion: Patients were divided into 4 groups and were enrolled in the study:}

1. Group I: Oncology Patients.

2. Group II: Cardiac Patients.

3. Group III: GIT Patients.

4. Group IV: Miscellaneous Patients.

\section{Inclusion criteria:}

All patients with a CVC, patients on CPAP and patients on mechanical ventilator were eligible for inclusion if they had a laboratory-confirmed 
BSI diagnosed after evaluation in our hospital and patients with multiple distinct episodes of BSI were included more than once. Cases were including those patients with monomicrobial and polymicrobial BSI.

Exclusion criteria: Patients with blood culture results positive for mycobacteria or viruses, patients discharged from the emergency department without hospitalization and patients died within 24 hours of admission.

\section{Sampling technique and sample size determination:}

A) Sample size: Assuming that total number of pediatric patients attending PICU at General Zagazig Hospital, was 120, and the prevalence of Gram +ve infection was $60 \%$. So, the sample size is 90 , CI $95 \%$ and power of test $80 \%$.

B) Sampling technique: The participants were chosen by systematic random sampling from patients admitted to PICU at General Zagazig Hospital.

\section{All patients included in the study were subjected to the following: \\ Sample collection:}

$2.5 \mathrm{ml}$ blood was collected under complete aseptic precautions. Aerobic and anaerobic culture was done using Vitek MS and BACT/ALERT. Positive blood culture results was identified among patients with a CVC, patients on CPAP and patients on mechanical ventilator by review of clinical microbiology laboratory records.

The ambulatory medical record and blood culture results of all patients with positive blood culture results in the context of a CVC had been reviewed to identify those who met our study definition for laboratory confirmed BSI. Study patients had been randomly selected from all eligible patients over the study period by use of a computergenerated random-number table.

The hospital medical records of patients selected for study inclusion had been reviewed for the following additional information: demographic characteristics, visit location, comorbid medical conditions, catheter type, number of catheter lumens, date of catheter insertion, receipt of immunosuppressive medications (e.g., corticosteroids), use of growth factors or parenteral nutrition, receipt of antibiotics before diagnosis, recent health care visits or hospital discharge, recent BSI, signs and symptoms at initial evaluation, results of initial laboratory evaluation, and blood culture results.
Data were collected with regard to clinical outcomes, including hospital disposition, date of discharge, and death during hospitalization, date of catheter removal, and readmission for BSI.

Study definitions. A patient was considered to have laboratory-confirmed BSI if a recognized pathogen was isolated from 1 blood culture at the time of evaluation. In addition, a patient was also considered to have BSI if he or she had signs or symptoms of systemic illness (fever [temperature, $38^{\circ} \mathrm{C}$ ], chills, or hypotension) and the same commensal bacterial species (i.e., diphtheroids, Bacillus species, Propionibacterium species, coagulase-negative staphylococci, or micrococci) were isolated from 2 cultures of blood samples obtained during the initial evaluation.

A BSI was considered to be polymicrobial if 2 pathogens were isolated from cultures of blood samples obtained within $48 \mathrm{~h}$ after initial evaluation, irrespective of whether the isolates came from the same or different blood culture bottles.

Recent hospital discharge was defined as discharge from the hospital within 7 days before the date that the positive blood culture sample was drawn. This variable was used not to distinguish hospital- from home-acquired infections, but instead to reflect the likelihood of adherence to standard catheter care guidelines. Recent BSI was defined as confirmed BSI within 30 days before the date that the positive blood culture specimen was drawn. A patient was considered to have underlying gastrointestinal dysfunction if he or she had received a previous diagnosis of inflammatory bowel disease or short-gut syndrome or if he or she was receiving parenteral nutrition at the time of initial evaluation.

Ethical approval and written informed consent : An approval of the study was obtained from Zagazig University academic and ethical committee. A written consent of one of the parents was taken to conduct the study.

\section{Statistical analysis}

Statistical analysis was performed using SPSS version 25 software (IBM, 2017). Results were presented by tables and graphs. Continuous data were presented as mean, standard deviation, and range. Qualitative data were presented as frequencies and proportions and were compared by Fisher's exact test. $\mathrm{P}<0.05$ was considered significant.

\section{RESULTS}

Table (1) shows the demographic characters of the studied patients. 
Table (1): Demographic characters of studied patients

\begin{tabular}{|c|c|c|}
\hline Demographic characteristics & $\begin{array}{c}\text { Studied patients } \\
(\mathbf{n}=90)\end{array}$ & $\%(100)$ \\
\hline $\begin{aligned} \text { Age (months): } & \\
\bullet \quad & >\mathrm{IM} \leq 6 \text { month } \\
\bullet \quad & >6 \text { month }\end{aligned}$ & $\begin{array}{l}13 \\
77\end{array}$ & $\begin{array}{l}14.4 \\
85.6\end{array}$ \\
\hline $\begin{array}{lll}\text { Sex: } & \\
& \bullet & \text { Males } \\
& \bullet & \text { Females } \\
\end{array}$ & $\begin{array}{l}49 \\
41\end{array}$ & $\begin{array}{l}54.4 \\
45.6\end{array}$ \\
\hline $\begin{aligned} \text { Weight } & (\mathbf{K g}) \\
\bullet & \text { Mean } \pm \text { SD } \\
\bullet & \text { Range }\end{aligned}$ & $\begin{array}{l}14.1 \pm 15.8 \\
2-70\end{array}$ & \\
\hline $\begin{aligned} & \text { Resident: } \\
& \bullet \text { Rural } \\
& \bullet \text { Urban } \\
&\end{aligned}$ & $\begin{array}{l}70 \\
20\end{array}$ & $\begin{array}{l}77.8 \\
22.2\end{array}$ \\
\hline
\end{tabular}

This table (2) shows that polymicrobial infection was associated with intubation.

Table (2): Risk factors of poly microbial and monomicrobial infection

\begin{tabular}{|l|c|c|c|c|c|}
\multirow{2}{*}{\multicolumn{1}{c|}{ Risk factors }} & \multicolumn{2}{c|}{$\begin{array}{c}\text { Monomicrobial } \\
(\mathbf{n}=\mathbf{7 0})\end{array}$} & \multicolumn{2}{c|}{$\begin{array}{c}\text { Polymicrobial } \\
(\mathbf{n = 4 )}\end{array}$} & \multirow{2}{*}{ P } \\
\cline { 2 - 5 } & No. & $\mathbf{\%}$ & No. & \% & \\
\hline Total parenteral nutrition (TPN) & 25 & 33.4 & 2 & 2.7 & $>0.05$ \\
\hline Intubation & 16 & 21.6 & 2 & 2.7 & $>0.05$ \\
\hline H2 blocker & 69 & 93.2 & 4 & 5.4 & $>0.05$ \\
\hline CVC & 21 & 28.4 & 2 & 2.7 & $>0.05$ \\
\hline CHD & 17 & 23 & 2 & 2.7 & $>0.05$ \\
\hline Steroids given to the patients & 52 & 70.3 & 1 & 1.4 & 0.033 \\
\hline Double lumen catheter & 6 & 12.5 & 1 & 1.4 & $>0.05$ \\
\hline Urinary catheter & 13 & 17.6 & 2 & 2.7 & $>0.05$ \\
\hline Congenital malformations & 29 & 39.2 & 3 & 4.1 & $>0.05$ \\
\hline
\end{tabular}

Table 3 shows that the first important risk factor for polymicrobial infection was steroids which were given to the patients, CVC, followed by, intubation and double lumen catheter.

Table (3): Risk assessment of polymicrobial sepsis

\begin{tabular}{|l|c|}
\multicolumn{1}{|c|}{ Risk factors } & Odds ratio $(95 \%$ CI $)$ \\
\hline Total parenteral nutrition (TPN) & $0.476(0.249-0.920)$ \\
\hline Intubation & $2.1(0.07-30.0) *$ \\
\hline H2 blocker & $0.968(0.958-1.014)$ \\
\hline CVC & $2.33(0.311-17.7)$ \\
\hline CHD & $0.486(0.168-4.1)$ \\
\hline Steroids given to the patients & $2.971(0.541-16.31)$ \\
\hline Double lumen catheter & $1.46(0.147-15.2)$ \\
\hline Urinary catheter & $0.371(0.124-4.37)$ \\
\hline Congenital malformations & $0.252(0.134-1.045)$ \\
\hline Stict
\end{tabular}

* Statistically significant

Table 4 shows the characters of the septic area. 
Table (4): characters of septic cases

\begin{tabular}{|l|c|c|}
\hline NICU admission & $\mathbf{N}(\mathbf{9 0})$ & $\mathbf{\%}$ \\
\hline$\quad$ Yes & & $\mathbf{5 3 . 3}$ \\
- No & $\mathbf{4 8}$ & $\mathbf{4 6 . 7}$ \\
\hline Type of patient & & \\
- Cardiac & 60 & 66.7 \\
- Oncology & 4 & 4.4 \\
- Gastroenterology & 2 & 2.2 \\
- Miscellaneous & 24 & 26.7 \\
\hline Cause of admission to PICU & & \\
- Respiratory & 58 & 64.4 \\
- CNS & 18 & 20 \\
- Renal & 14 & 15.6 \\
\hline
\end{tabular}

\section{DISCUSSION}

During our study period, 90 children with suspected sepsis using clinical criteria were enrolled, only $74(82.2 \%)$ were confirmed to have blood stream infection by using blood culture.

This rate is comparable to rates reported in other developing African and Asian countries as Bangladesh (34.88\%) had positive blood culture ${ }^{(10)}$, Ethiopia (44.7\%) (11), and Nigeria (45\%) ${ }^{(12)}$. However, negative blood culture was about $26 \%$ of all sepsis could be due to anaerobes. Furthermore, the etiological agents may not be isolated by media used as viral (e.g., rubella, cytomegalovirus), protozoal (e.g., toxoplasma gondii, and Treponema pallidum) pathogen ${ }^{(13)}$.

We observed that monomicrobial bloodstream infections (MBSIs) were associated with an increased mortality $36.5 \%$ compared to polymicrobial (P) BSI death rate was $2.7 \%$ although these differences were not statistically significant. These high rates were not agreed with Pammi $\boldsymbol{e t}$ al. (14) death rate of PBSIs was $47.1 \%$ in comparison to MBSIs was $19.6 \%$ and BSI-related death occurred in $16 \%$ of cases of polymicrobial BSI and $8 \%$ of cases of monomicrobial BSI. They also disagreed with Gupta et al. ${ }^{(15)}$ who reported no difference in mortality rate; may be due to they did not discriminate between contaminant and true pathogen, based on the clinical features. This could be the reason for not studying a detailed analysis of cause of death in the polymicrobial group, as to whether the death occurred because of the underlying disease (s) or could be attributed to multiple invading organisms.

Regarding demographics in our study; mean weight was $14.1 \pm 15.8$ and in the polymicrobial group was $4.25 \pm 3.2$ and in the monomicrobial group $13.8 \pm 18.1$. The age in the polymicrobial group (5.4\%) was $>1 \mathrm{M} \leq 6$ month and in $(82.4 \%)$ was $>6$ month monomicrobial groups.

In our study polymicrobial infection was higher in those from rural area than in those from urban area. In our study there was a statistically significant difference between monomicrobial and polymicrobial sepsis in chronological age, as polymicrobial infection was associated with younger chronological age than monomicrobial infection. A possible explanation for the association between polymicrobial BSIs and age could be the presence of different groups of underlying illnesses with diff erent treatments, immune status and different types of catheters. However, none of the other variables, (with the exception of venous implanted ports) showed a relation with the frequency of polymicrobial BSIs. Furthermore, younger children are less aware of the vulnerability of their IVD, resulting in risky behavior. Younger children also have different skin microbiota with fecal contamination, which might result in a higher risk of getting infected by polymicrobial BSI than older children ${ }^{(16)}$.

This was disagreed with the study of Onland et al. ${ }^{(16)}$, which recorded that PBSI and MBSI had different demographics and reported that mean age of studied group was $4.1(1.9-12.5)$ years.

In this study $54.4 \%$ were male, and $45.6 \%$ was female and age of studied groups was $85.6 \% \geq 6$ month. This coped with the study of Onland $\boldsymbol{e t}$ al. (16) who studied pediatric patients with intravascular devices: polymicrobial blood stream infections and risk factors in which $60.6 \%$ were male, and $39.4 \%$ was female. Also we observed that predisposing factors to polymicrobial infection were in, recipient of $\mathrm{H} 2$ blockers, and CHD.

The first important risk factor for polymicrobial infection in PICU sepsis was, steroids use by patients, CVC, intubation, and double lumen 
catheter. This agreed with Sutter et al. ${ }^{(17)}$ who reported that risk factors for BSI (i.e., use of total parenteral nutrition, intralipids, H2-blockers) had to be present at the time the culture-positive blood sample was obtained to qualify as a risk factor. Severe underlying conditions included as risk factors only if diagnosis preceded the onset of BSI, consisted of any major congenital anomaly (e.g., congenital heart disease, gastroschisis, jejunal atresia, etc.) and/or acquired life-threatening conditions (e.g., necrotizing enterocolitis, major end organ failure, intracranial hemorrhage, and/or respiratory failure of any cause) and their associated interventions. Also they reported that risk factors for polymicrobial infections in children and adults include the presence of a central venous catheter, administration of parenteral nutrition, gastrointestinal pathology, especially short gut syndrome, use of broadspectrum antibiotics and immune-suppression.

In this study, $53.3 \%$ of our patients had previous NICU admission, $66.7 \%$ have cardiac disease and $64.4 \%$ have respiratory cause of admission to PICU. Also clinical manifestations regarding PBSIs respiratory $(75 \%)$, renal failure $(25 \%)^{(15)}$. Reported that clinical manifestations regarding PBSIs were general $(88.9 \%)$, respiratory (75\%), GIT (80.6\%), hematological (47.2\%) and neurological (22.2\%) in comparison to MBSIs were general $(92.2 \%)$, respiratory $(82.8 \%)$, GIT $(70.3 \%)$, hematological $(57.8 \%)$ and neurological (23.4\%). Also Rostaing and Malvezzi ${ }^{(18)}$ reported that with increasing doses the rate of occurrence of infectious complications increased in patients given corticosteroids as well as in patients given placebo, a finding suggesting that not only the corticosteroid but also the underlying disease state account for the steroid-associated infectious complications observed in clinical practice. The clinical data regarding PBSIs was limited due to most of the studies were a retrospective, observational, case-control studies and bloodstream episodes were identified from the clinical microbiology database ${ }^{(\mathbf{1 4})}$.

We observed that incidence of polymicrobial infection of sepsis was associated more with Gram negative organisms than Gram positive organisms and fungi. The isolated Gram negative organisms were $6.9 \%$ klebsiella 1case $(1.4 \%)$, Serratia marcescens 1 case $(1.4 \%)$ and (4.1\%) Pseudomonas. The isolated Gram positive organisms (CONS) in 2 cases $2.8 \%$ and the isolated fungi was candida in one case $1.4 \%$ of PBSIs.

In comparison to monomicrobial infections in our study; incidence of MBSIs was predominately associated with Gram negative organisms $35.8 \%$ especially [klebsiella 9 cases $(12.2 \%)$, Serratia marcescens 5 cases $(6.8 \%)$, E. Coli 8 case $(10.8 \%)$, Acinetobacter baumannii complex 2 case $(2.7 \%)$, Pseudomonas 2 case $(2.7 \%)$ ] compared to Gram positive organisms 54\% [CONS 32 cases (43\%), staphylococcus coccus aureus 6 cases $(8.1 \%)$, Streptococcus pneumoni 2 case (2.7\%)] and candida 4 case $(5.5 \%)$. CONS isolated in MBSIs were Staph haemolyticus in 15 cases (20.3\%), staph epidermis in 4 cases $(5.4 \%)$, staph hominis in 11 cases $(14.9 \%)$ and Staphylococcus saprophytic in 2 cases $(2.7 \%)$. Similar findings reported in Faix and Kovarik ${ }^{(19)}$ study, where Gram-negative organisms constituted nearly three fifth $(59.8 \%)$ of the PBSIs, which was significantly higher percentage than Gram-positive organisms.

Also, Tsai et al. ${ }^{(20)}$ reported that, Gramnegative organisms constituted nearly three-fifth $(59.8 \%)$ of the case group organisms, which was a significantly higher percentage than Gram-positive organisms $(59.8 \%$ vs. $39.1 \%)$. Gram-negative organisms were also more common in PBSIs episodes than MBSIs. In 33 (73.3\%) episodes of PBSIs, at least one of the causative organisms was Gram-negative bacilli, and 22 (48.9\%) episodes of PBSIs were caused by two or more Gram-negative organisms. Only one PBSI episode had Candida spp.

On the contrary in another study, Grampositive cocci were recovered from culture in 81 (77\%) of the 105 cases of polymicrobial BSIs. Coagulase negative staphylococcus (CoNS) were the most common organisms recovered overall and were present in 47 cases (43\%). Species of Enterococcus were present in 46 cases (44\%); Gram-negative rods were present in 57 cases (54\%); Escherichia coli was the most common gram negative rod species recovered from culture and was present in 20 cases (19\%). Fungi were present in 19 cases of polymicrobial BSIs (18\%); Candida albicans was the causative agent in 13 cases $(12 \%)^{(21)}$.

In our study; all cases of PBSIs were caused by two bacterial pathogens, and identified from different blood specimens. This agreed with Tsai et al. ${ }^{(20)}$ who found that the majority of PSBIs were caused by two bacterial pathogens (43/45, 95.6\%), and only two PSBI episodes had three pathogens identified.

Also Pammi et al. ${ }^{(14)}$ reported that, the isolated organisms of PBSI were from different blood specimens. Disagreed with Pammi et al. ${ }^{(14)}$; Gupta et al. (15) and Tsai et al. (20) defined polymicrobial infections as multiple pathogens isolated from a single blood specimen, which may account for the lower incidence of polymicrobial infections.

\section{CONCLUSION}

Steroids given to the patients, endotracheal intubation, CVC, CHD and $\mathrm{H} 2$ blocker were the 
most common risk factors for polymicrobial infection in PICU.

\section{REFERENCES}

1. Bagchi S, Watkins J, Pollock D et al. (2018): State health department validations of central lineassociated bloodstream infection events reported via the National Healthcare Safety Network. Am J Infect Control, 46(11):1290-1295.

2. Zingg W, Marschall J (2018): Surveillance Associated and prevention of infections with vascular catheters. Practical healthcare epidemiology, Pp. 162. DOI: https://doi.org/10.1017/9781107153165.016

3. Chesshyre E, Goff Z, Bowen A et al. (2015): The prevention, diagnosis and management of central venous line infections in children. Journal of Infection, 71, S59-S75.

4. Kreuziger L, Jaffray J, Carrier M (2017): Epidemiology, diagnosis, prevention and treatment of catheter-related thrombosis in children and adults. Thrombosis Research, 157: 64-71.

5. Mvalo T, Eley B, Bamford C et al. (2017): Blood stream infections in oncology patients at Red Cross War Memorial Children's Hospital, Cape Town. Int J Infect Dis., 77:40-47.

6. Elfassy S, Kassam Z, Amin F et al. (2015): Epidemiology and risk factors for bloodstream infections in a home parenteral nutrition program. Journal of Parenteral and Enteral Nutrition, 39(2): 147-153.

7. Taylor R, Shekerdemian L (2016): Avoidance of hospital-acquired infections in pediatric cardiac surgical patients. Pediatric Critical Care Medicine, 17(8): 279-286.

8. Downes K, Metlay J, Bell $L$ et al. (2008): Polymicrobial bloodstream infections among children and adolescents with central venous catheters evaluated in ambulatory care. Clinical Infectious Diseases, 46(3): 387-394.

9. Barlam T, Cosgrove S, Abbo L et al. (2016): Implementing an antibiotic stewardship program: guidelines by the Infectious Diseases Society of America and the Society for Healthcare Epidemiology of America. Clinical Infectious Diseases, 62(10): 51-77.
10. Ahmed A, Chowdhury M, Hoque S et al. (2002): Clinical and bacteriological profile of neonatal septicemia in a tertiary level pediatric hospital in Bangladesh. Indian Pediatrics, 39(11): 1034-1039.

11. Shitaye D (2008): Neonatal sepsis: Bacterial etiologic agents and their antibiotic susceptibility pattern in Tikur Anbessa University Hospital, Addis Ababa, Ethiopia. Addis Ababa University Journal. http://etd.aau.edu.et/handle/123456789/5116

12. Meremikwu M, Nwachukwu $C$, Asuquo A et al. (2005): Bacterial isolates from blood cultures of children with suspected septicemia in Calabar, Nigeria. BMC Infectious Diseases, 5: 110-115.

13. Shehab El-Din E, Adel El-Sokkary M, Bassiouny M et al. (2015): Epidemiology of neonatal sepsis and implicated pathogens: A study from Egypt. Bio Med Research International, 509484: 11. http://dx.doi.org/10.1155/2015/509484.

14. Pammi M, Zhong D, Johnson Y et al. (2014): Polymicrobial bloodstream infections in the neonatal intensive care unit are associated with increased mortality: a case-control study. BMC Infectious Diseases, 14(1): 390-95.

15. Gupta P, Kumhar G, Kaur G et al. (2005): Clinical significance of polymicrobial bacteremia in newborns. J Pediatric Child Health, 41:365-368.

16. Onland W, Pajkrt D, Shin C et al. (2011): Pediatric patients with intravascular devices: polymicrobial bloodstream infections and risk factors. Journal of Pathogens, 3:826169-73.

17. Sutter D, Stagliano D, Braun L et al. (2008): Polymicrobial bloodstream infection in pediatric patients: risk factors, microbiology, and antimicrobial management. The Pediatric Infectious Disease Journal, 27(5): 400-405.

18. Rostaing L, Malvezzi P (2016): Steroid-based therapy and risk of infectious complications. PLoS Medicine, 13(5): 2025-33.

19. Faix R, Kovarik S (1989): Polymicrobial sepsis among intensive care nursery infants. J Perinatol., 9(2):131-136.

20. Tsai M, Chu S, Hsu J et al. (2014): Risk factors and outcomes for multidrug-resistant Gram-negative bacteremia in the NICU. Pediatrics, 133(2): 322-9.

21. Ivády B, Kenesei É, Tóth-Heyn $P$ et al. (2016): Factors influencing antimicrobial resistance and outcome of Gram-negative bloodstream infections in children. Infection, 44(3): 309-321. 